\title{
Early manifestations of renal disease in patients with tuberous sclerosis complex
}

\author{
Laura Malaga-Dieguez' \\ Robert Spencer' \\ Laura J Pehrson' \\ Suzanne Vento' \\ Kimberly Menzer ${ }^{2}$ \\ Orrin Devinsky² \\ Howard Trachtman' \\ 'Division of Pediatric Nephrology, \\ ${ }^{2}$ Department of Neurology, \\ Comprehensive Epilepsy Center, New \\ York University School of Medicine, \\ New York, NY, USA
}

Correspondence: Laura Malaga-Dieguez Division of Pediatric Nephrology, Comprehensive Epilepsy Center, New York University School of Medicine, 403 E 34 ${ }^{\text {th }}$ St., Room 104, New York, NY 10016, USA

Tel + I $64650 \mid 2669$

$\mathrm{Fax}+\mathrm{I} 2122634053$

Email laura.malaga-dieguez@nyumc.org
This article was published in the following Dove Press journal: International Journal of Nephrology and Renovascular Disease 2 May 2017

Number of times this article has been viewed

Objectives: Renal manifestations are the second most significant cause of morbidity and mortality in patients with tuberous sclerosis complex (TSC), and include renal cysts, angiomyolipomas, fat-poor lesions, and malignant tumors. These lesions begin in childhood and often lead to chronic kidney disease (CKD). Little is known on the incidence of early modifiable risk factors of CKD, such as proteinuria and hypertension, or subtle decreases in glomerular filtration rate that correspond to the early stages of CKD in children with TSC. The impact of genotype on these early manifestations of CKD has not been investigated.

Design: Retrospective chart review of 84 children and young adults with TSC.

Measurements: This study assessed the prevalence of hypertension, renal impairment, and proteinuria, as well as the genotype-phenotype correlations.

Results: Children and young adults with TSC2 mutations had a significantly higher rate of renal lesions, hypertension ( $36 \%$ vs 14\%), and decreased renal function than those with TSC1 mutations.

Conclusion: On the basis of estimated glomerular filtration rate and blood pressure, our findings are consistent with the hypothesis that TSC2 mutations are associated with more severe early renal involvement in children. There is a compelling need for close collaboration of nephrologists and neurologists to provide care to pediatric patients with TSC to improve screening and management of early manifestations of renal disease.

Keywords: tuberous sclerosis complex, chronic kidney disease, hypertension, proteinuria, estimated glomerular filtration rate

\section{Introduction}

Tuberous sclerosis complex (TSC) is an autosomal dominant genetic disorder in a third of cases and results from de novo mutations in the other two-thirds of cases. TSC is associated with cognitive and behavioral changes, and characterized by predisposition to nonmalignant lesions throughout the body, including the kidneys. ${ }^{1}$ Renal manifestations are the second most significant cause of morbidity and mortality in patients with TSC, and include renal cysts, angiomyolipomas, fat-poor lesions, and malignant tumors. ${ }^{2-4}$ These lesions begin in infancy and can become large enough that they can encroach on normal renal tissue, resulting in hypertension and chronic kidney disease (CKD). 5,6

TSC is caused by mutations in either the TSC1 gene, located on chromosome 9 and encoding hamartin, or the TSC2 gene, located on chromosome 16 and encoding tuberin. Disease-causing mutations can be identified by mutation analysis in $60 \%-89 \%$ of patients who meet the diagnostic criteria for TSC, including $\sim 50 \%$ with mutations in 
TSC2 and $17 \%$ with mutations in TSC $1 .^{7-9}$ Mutations in TSC2 tend to result in a more severe clinical phenotype than those in TSC $1,{ }^{10}$ including the renal involvement. ${ }^{11,12}$ In addition, the gene for autosomal dominant polycystic kidney disease 1 (PKD1) lies adjacent to the TSC2 gene on chromosome 16 , and 'contiguous gene deletions' can result in multiple renal cystic lesions, similar to those seen in polycystic kidney disease. ${ }^{13,14}$ This condition is detectable in infancy or early childhood and generally leads to renal insufficiency in the late teens to early $20 \mathrm{~s}^{15}$

The renal disorders in TSC are well defined, with the exception of early manifestations of CKD such as proteinuria and hypertension that may be amenable to modification. Because the incidence of early stages of CKD is often asymptomatic, they are not well characterized in these patients. Even less is known about the effect of genotype in these early markers of kidney disease and progression.

This study investigates the presence of proteinuria, reduced glomerular filtration rate (GFR) and hypertension in a cohort of pediatric and young adults with TSC, and examines whether genotype correlates with phenotype.

\section{Patients and methods}

We conducted a single-center, retrospective review of children and young adults (aged $<25$ years of age) who were followed by the Tuberous Sclerosis Complex Center at NYU School of Medicine. This study was approved by the NYU School of Medicine's Institutional Review Board (IRB). The information was recorded in such a manner that subjects could not be identified, directly or through identifiers linked to the subjects. Therefore, a waiver of authorization for consent was granted by the IRB. The medical record was reviewed to ensure that all subjects had confirmed mutational analysis for TSC. Those without genetic testing were excluded from the study; pregnant patients, patients with primary or secondary causes of nephrotic syndrome, patients with other secondary causes of hypertension, and patients with severe pulmonary manifestations of TSC, specifically those requiring highfrequency oscillation, were also excluded from all analysis. Anthropometric variables and demographic data including age, race, and gender of the children and young adults were recorded. In all patients, the average of office blood pressure measurements on their last three consecutive visits was used for analysis. Guidelines from "The fourth report on the diagnosis, evaluation, and treatment of high blood pressure in children and adolescents" child or adolescent had hypertension. Diagnostic test results (eg, renal ultrasonography, abdominal magnetic resonance imaging) and laboratory parameters were also tabulated. Proteinuria was estimated using urinalysis when available. Proteinuria on dipstick was reported as negative, $1+$ or trace $(30 \mathrm{mg} / \mathrm{dL}), 2+(100 \mathrm{mg} / \mathrm{dL}), 3+(300 \mathrm{mg} / \mathrm{dL})$, or $4+$ $(1000 \mathrm{mg} / \mathrm{dL})$. None and trace urinary protein were classified as no proteinuria and remaining readings $(2+, 3+$, and $4+)$ as proteinuria. The GFR was estimated using the modified Schwartz formula. ${ }^{17}$

Subjects were assigned to one of three groups based on the results of their genetic testing: 1) TSC1 mutation, 2) TSC2 mutation, or 3 ) unidentified mutation. Results were expressed as absolute numbers, proportions, means with standard deviations or medians with range. Nominal variables were analyzed for associations using chi-square test and crude (unadjusted) odds ratio were calculated where appropriate. Interval variables were analyzed using Student's $t$-test for normally distributed variables and Wilcoxon's rank sum test for non-normal distributions. All statistical tests were done using GraphPad Prism version 6.05 for Windows (GraphPad Software, La Jolla CA, USA). $p<0.05$ was taken as significant.

\section{Results}

A total of 84 charts were reviewed as part of this study (Figure 1). Of these, 49 were excluded due to no documented mutational analysis (36) or age $>25$ years at the time of diagnosis (13). Thus, a total of 35 patients fulfilled the inclusion criteria for the study. Seven of these patients had a documented TSC1 mutation, 22 had a documented TSC2 mutation, 3 had a non-TSC1 or TSC2 documented mutation ( 1 chromosome 6 mutation, 2 unidentifiable mutations) while 3 patients had unknown mutations by standard genotyping even though there was a characteristic clinical diagnosis of TSC. The greater prevalence of TSC 2 over TSC1 mutations was consistent with prior studies. ${ }^{9,11}$ The patients were 1-30 years old at the time of study, with a median age of 8 years and an average age of 11 years. Sporadic (de novo) cases accounted $71 \%$ of TSC1 and $52 \%$ of TSC 2 patients. Our study population characteristics were similar to those of published case series in terms of distribution of TSC1 and TSC 2 across age groups and gender (Table 1). Also comparable to large series, ${ }^{11}$ there was increased severity of TSC2, compared with TSC1, disease in multiple organs including the kidneys: $68 \%$ of TSC 2 versus $50 \%$ of TSC 1 patients had renal involvement (Figure 2).

\section{Hypertension}

Based on office blood pressure measurements, only one patient with TSC1 was hypertensive, while hypertension was 


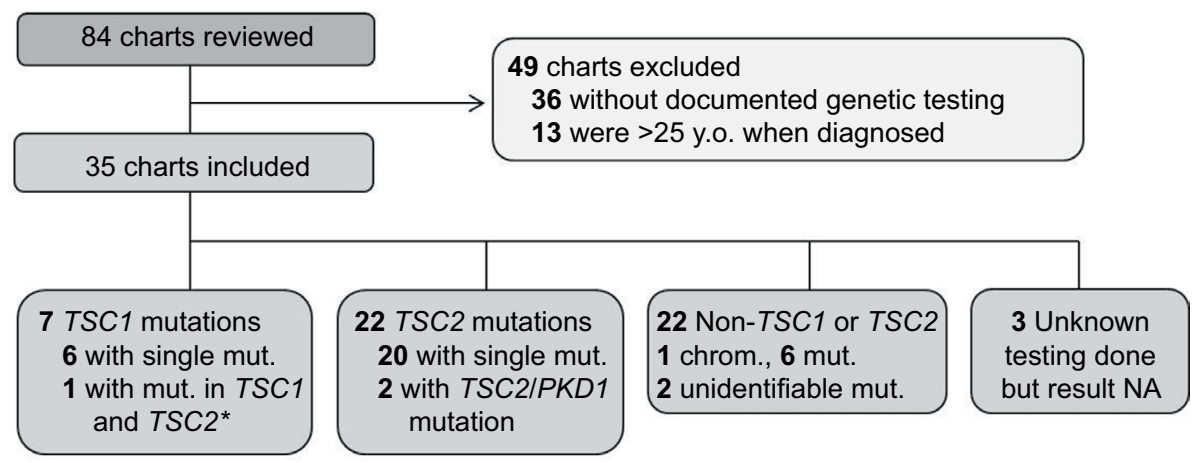

Figure I CONSORT diagram of study participants.

Note: *Only TSCI mutations are pathogenic.

Abbreviations: chrom., chromosome; mut., mutation; NA, not applicable; PKDI, polycystic kidney disease I; TSC, tuberous sclerosis complex; y.o., years old.

Table I Baseline demographics and clinical characteristics by mutation

\begin{tabular}{|c|c|c|c|c|}
\hline Variables & $\begin{array}{l}\text { TSCI } \\
(n=7)\end{array}$ & $\begin{array}{l}\text { TSC2 } \\
(n=22)\end{array}$ & $\begin{array}{l}\text { Non-TSCI-Non- } \\
\text { TSC2 mutations } \\
(n=3)\end{array}$ & $\begin{array}{l}\text { Unknown } \\
(n=3)\end{array}$ \\
\hline Age, mean $( \pm S D)$ & $9( \pm 4.2)$ & II ( \pm 9.2$)$ & $17( \pm 10)$ & $19( \pm 13)$ \\
\hline Male, N (\%) & $6(86)$ & $8(36)$ & $2(67)$ & I (33.3) \\
\hline \multicolumn{5}{|l|}{ Race, N (\%) } \\
\hline White & $6(86)$ & $18(82)$ & $3(100)$ & $3(100)$ \\
\hline Black & 0 & $3(13.5)$ & 0 & 0 \\
\hline Asian & I (I4) & I (4.5) & 0 & 0 \\
\hline $\begin{array}{l}\text { Family history of } \\
\text { TSC }^{\text {a }}\end{array}$ & $2(28.5)$ & $10(48)$ & $2(67)$ & NA \\
\hline Renal involvement ${ }^{\mathrm{b}}$ & $3(50)$ & $13(68)$ & I (33) & I (33) \\
\hline $\begin{array}{l}\text { Neurological } \\
\text { manifest }\end{array}$ & $7(100)$ & $19(86)$ & $2(67)$ & $3(100)$ \\
\hline $\begin{array}{l}\text { Taking mTOR } \\
\text { inhibitor }\end{array}$ & $2(28.5)$ & $3(13.6)$ & I (33) & I (33) \\
\hline
\end{tabular}

Notes: a No family history available for an adopted child with TSC2. ${ }^{\text {bNo renal }}$ imaging available for I of the TSCI patients and 3 of the TSC2 patients.

Abbreviations: SD, standard deviation; TSC, tuberous sclerosis complex; mTOR, mechanistic target of rapamycin; NA, not applicable.

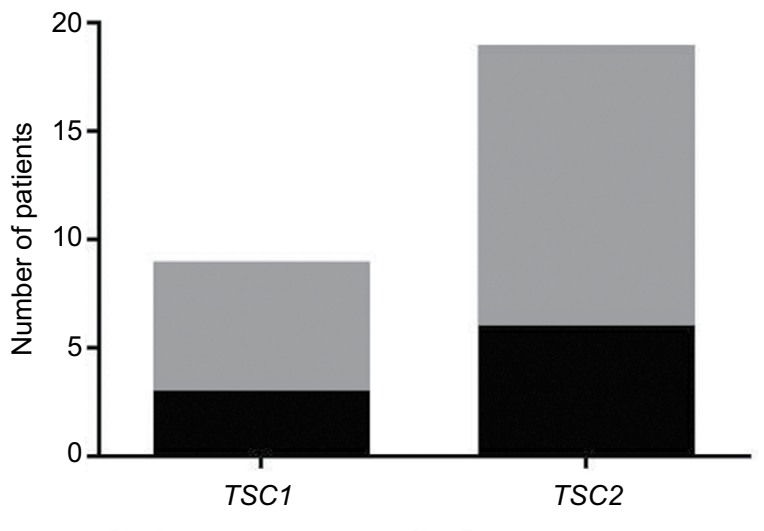

Renal involvement No renal involvement

Figure 2 Number of patients with TSCI or TSC2 with renal involvement. Abbreviation: TSC, tuberous sclerosis complex.

found in 8 out of 22 patients with TSC2 (36\%; Figure 3). The TSC1 patient with hypertension did not have renal lesions identified on imaging, while all but one of the hypertensive

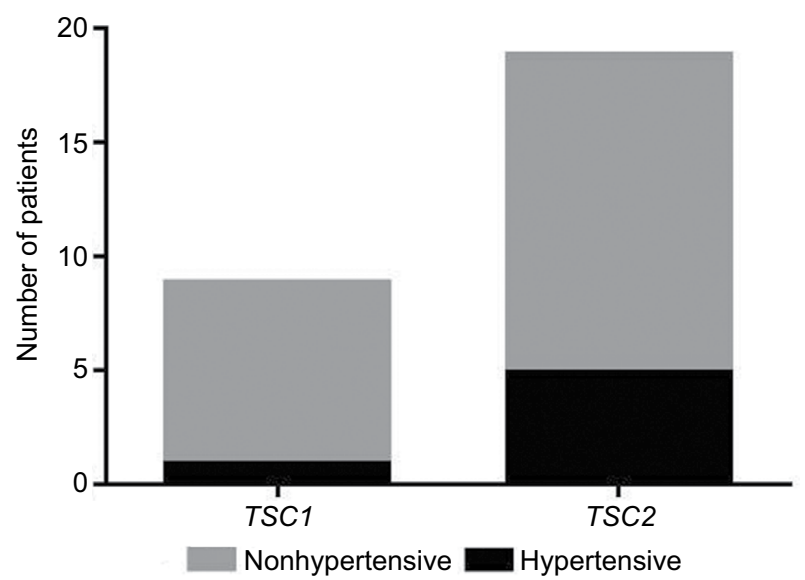

Figure 3 Number of patients with TSCI or TSC2 with hypertension. Abbreviation: TSC, tuberous sclerosis complex.

TSC2 patients had significant renal lesions identified. None of the hypertensive patients identified was receiving angiotensin-converting enzyme inhibitor or other antihypertensive therapy.

\section{Proteinuria}

Urinalysis was not performed routinely. Only 2 out of 7 patients from the TSC1 and 12 out of 22 patients from the TSC2 group had urine specimens, and no significant proteinuria was detected in any patient. The patients with urine specimens had either been referred to pediatric nephrology for follow-up and the urine specimen was obtained by the pediatric nephrologist, had a urinalysis obtained during a hospital admission for a disease complication, or were females in reproductive age receiving intense pharmacological intervention. Only two patients routinely followed by pediatric nephrology had routine spot urine protein and creatinine (Upr/cr) quantitation. The $\mathrm{Upr} / \mathrm{cr}$ in these two samples was $<0.2 \mathrm{mg} / \mathrm{mg}$. 


\section{Glomerular filtration rate}

All but one patient with TSC1 mutations had at least one serum creatinine documented in the electronic medical record. The estimated glomerular filtration rate (eGFR) was $\geq 90 \mathrm{~mL} / \mathrm{min} / 1.73 \mathrm{~m}^{2}$ in all of them (100\%). However, only 15 patients with a TSC2 mutation had both serum creatinine levels and height documented in the chart for appropriate eGFR calculation. Three of these patients had a low eGFR with two having mild renal impairment and one having moderate renal impairment (Table 2). Serum creatinine was within the normal range in 12 of $15(80 \%)$ patients with TSC2 mutations (Table 2 and Figure 4). Older patients were more likely to have a low eGFR compared with the younger children. A 7-year-old patient with a TSC2 mutation had a severe episode of nephrotoxic acute kidney injury with peak serum creatinine of $3.3 \mathrm{mg} / \mathrm{dL}$ (eGFR at the time $<30 \mathrm{~mL} /$ $\min / 1.73 \mathrm{~m}^{2}$ ) that returned to baseline prior from discharge from the hospital.

\section{Discussion}

This study shows a higher prevalence of renal abnormalities in children and young adults with TSC2 mutations, compared with those with TSC1 mutations, with significant hypertension in $36 \%$ and a low eGFR in $20 \%$ in the TSC2 group. Proteinuria was not consistently measured, but was absent in all patients with urine specimens. However, because of

Table 2 Estimated GFRs by mutation

\begin{tabular}{lll}
\hline & TSCI, n=6 (\%) & TSC2, n= I5 (\%) \\
\hline Normal GFR $(\geq 90)$ & $6(100)$ & $12(80)$ \\
Stage I CKD (60-89) & 0 & $2(13.3)$ \\
Stage 2 CKD (30-59) & 0 & $1(6.6)$ \\
\hline
\end{tabular}

Abbreviations: CKD, chronic kidney disease; GFR, glomerular filtration rate; TSC, tuberous sclerosis complex.

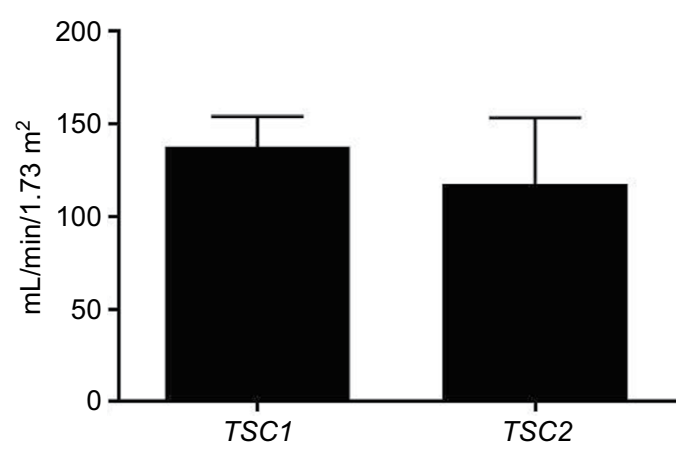

Figure 4 Estimated glomerular filtration rate in patients with $T S C I$ and TSC2. Abbreviation: TSC, tuberous sclerosis complex. limited testing, we cannot comment on the relative severity of proteinuria in the two TSC genotypes.

Current international guidelines for surveillance and management of TSC recommend obtaining abdominal imaging at the time of diagnosis ${ }^{18}$ and suggest that magnetic resonance imaging is superior to ultrasound and computed tomography. Magnetic resonance imaging represents the preferred evaluation modality for angiomyolipomas (especially fat-poor lesions). This appears to be consistent in our study - almost all our patients had one or more renal imaging studies. In addition to imaging, the consensus guidelines suggest assessment of blood pressure and eGFR at diagnosis and annually after that. Clinical guidelines recommend adequate blood pressure control. However, we found a high prevalence of hypertension that was often missed in the clinical setting. It appears that in our population, age-specific criteria for children were not easily accessible and utilized. Patients with hypertension were not treated or referred for further management by pediatric nephrology.

Similar findings apply to the eGFR. Even when the Schwartz formula is incorporated into the medical record system, subtle decreases in eGFR were often missed.

There is no recommendation in the guidelines on the evaluation of proteinuria. While we did not find any patient with significant proteinuria, this may reflect the low rate of screening. Proteinuria is an essential parameter in the detection of early kidney damage that is amenable to intervention, namely use of an angiotensin-converting enzyme inhibitor or angiotensin receptor blocker to slow progression of CKD. Clinical trials are needed to determine if this intervention is renoprotective and can alter the rate of progression of CKD in patients with TSC.

\section{Study limitations}

Our study is a small single-center study with a heterogeneous group of children and young adults with TSC followed at an internationally recognized center of excellence for TSC. However, there are limited data about the prevalence of hypertension, proteinuria, and alterations in GFR in the literature. Early findings of renal disease have not received much attention. A finding of increased prevalence of hypertension and decreases in GFR may be important and clinically useful. In light of our finding that proteinuria is not being routinely monitored, large-scale multicenter prospective studies with long-term follow-up could yield more comprehensive reports about the prevalence of hypertension, proteinuria, and rate of decline of renal function and its influence in progression of CKD. 


\section{Conclusion}

On the basis of eGFR and blood pressures, our findings are consistent with the hypothesis that $T S C 2$ mutations are associated with earlier and more severe renal involvement in children. There is a great need for closer collaboration between nephrologists and neurologists in the provision of care for pediatric patients with TSC in order to foster adequate screening for early manifestations of renal disease. Furthermore, given the enormous progress in therapies for the complications of TSC, the mechanism of kidney involvement in TSC is shifting as well toward a multifactorial cause - not only related to genotype but also to the drugs used in the treatment of TSC and its complications. Multidisciplinary TSC centers may improve clinical care for children with TSC and foster natural history studies and clinical trials to improve therapy and outcome.

\section{Disclosure}

The authors report no conflicts of interest in this work.

\section{References}

1. Crino PB, Nathanson KL, Henske EP. The tuberous sclerosis complex. N Engl J Med. 2006;355(13):1345-1356.

2. Bernstein J, Robbins TO. Renal Involvement in tuberous sclerosis. Ann NY Acad Sci. 1991;615:36-49.

3. Ewalt DH, Sheffield E, Sparagana SP, Delgado MR, Roach ES. Renal lesion growth in children with tuberous sclerosis complex. $J$ Urol. 1998;160(1):141-145.

4. Rakowski SK, Winterkorn EB, Paul E, Steele DJ, Halpern EF, Thiele EA. Renal manifestations of tuberous sclerosis complex: incidence, prognosis, and predictive factors. Kidney Int. 2006;70(10):1777-1782.

5. O’Hagan AR, Ellsworth R, Secic M, Rothner AD, Brouhard BH. Renal manifestations of tuberous sclerosis complex. Clin Pediatr. 1996;35(10):483-489.
6. Henske EP. Tuberous sclerosis and the kidney: from mesenchyme to epithelium, and beyond. Pediatr Nephrol. 2005;20(7):854-857.

7. Au KS, Williams AT, Roach ES, et al. Genotype/phenotype correlation in 325 individuals referred for a diagnosis of tuberous sclerosis complex in the United States. Gene Med. 2007;9(2):88-100.

8. Nijda Y, Lawrence-Smith N, Lewis J, et al. Analysis of both TSC1 and TSC2 for germline mutations in 126 unrelated patients with tuberous sclerosis. Am J Hum Genet. 1999;65(4):A406-A406.

9. Jones AC, Shyamsundar MM, Thomas MW, et al. Comprehensive mutation analysis of TSC1 and TSC2-and phenotypic correlations in 150 families with tuberous sclerosis. Am J Hum Genet. 1999;64(5):1305-1315.

10. Curatolo P, Bombardieri R, Jozwiak S. Tuberous sclerosis. Lancet. 2008;372(9639):657-668.

11. Dabora SL, Jozwiak S, Franz DN, et al. Mutational analysis in a cohort of 224 tuberous sclerosis patients indicates increased severity of TSC2, compared with TSC1, disease in multiple organs. Am J Hum Genet. 2001;68(1):64-80.

12. Sancak O, Nellist M, Goedbloed M, et al. Mutational analysis of the TSC1 and TSC2 genes in a diagnostic setting: genotype-phenotype correlations and comparison of diagnostic DNA techniques in Tuberous Sclerosis Complex. Eur J Hum Genet. 2005;13(6):731-741.

13. Brook-Carter PT, Peral B, Ward CJ, et al. Deletion of the TSC2 and PKD1 genes associated with severe infantile polycystic kidney disease-a contiguous gene syndrome. Nat Genet. 1994;8(4):328-332.

14. Sampson JR, Maheshwar MM, Aspinwall R, et al. Renal cystic disease in tuberous sclerosis: role of the polycystic kidney disease 1 gene. $\mathrm{Am}$ J Hum Genet. 1997;61(4):843-851.

15. Longa L, Scolari F, Brusco A, et al. A large TSC2 and PKD1 gene deletion is associated with renal and extrarenal signs of autosomal dominant polycystic kidney disease. Nephrol Dial,Transplant. 1997;12(9):1900-1907.

16. National High Blood Pressure Education Program Working Group on High Blood Pressure in Children and Adolescents. The fourth report on the diagnosis, evaluation, and treatment of high blood pressure in children and adolescents. Pediatrics. 2004;114(2 Suppl 4th Report):555-576.

17. Schwartz GJ, MunozA, Schneider MF, et al. New equations to estimate GFR in children with CKD. J Am Soc Nephrol. 2009;20(3):629-637.

18. Krueger DA, Northrup H; International Tuberous Sclerosis Complex Consensus G. Tuberous sclerosis complex surveillance and management: recommendations of the 2012 International Tuberous Sclerosis Complex Consensus Conference. Pediatr Neurol. 2013;49(4):255-265.
The International Journal of Nephrology and Renovascular Disease is an international, peer-reviewed open access journal focusing on the pathophysiology of the kidney and vascular supply. Epidemiology, screening, diagnosis, and treatment interventions are covered as well as basic science, biochemical and immunological studies. The manuscript management system is completely online and includes a very quick and fair peer-review system, which is all easy to use. Visit http://www. dovepress.com/testimonials.php to read real quotes from published authors. 